\title{
Inverse Problems via the "Generalized Collage Theorem" for Vector-Valued Lax-Milgram-Based Variational Problems
}

\author{
H. Kunze, ${ }^{1}$ D. La Torre, ${ }^{2,3}$ K. Levere, ${ }^{1}$ and M. Ruiz Galán ${ }^{4}$ \\ ${ }^{1}$ Department of Mathematics and Statistics, University of Guelph, Guelph, ON, Canada N1G 2W1 \\ ${ }^{2}$ Department of Applied Mathematics and Sciences, Khalifa University, P.O. Box 127788, Abu Dhabi, UAE \\ ${ }^{3}$ Department of Economics, Management, and Quantitative Methods, University of Milan, 20122 Milan, Italy \\ ${ }^{4}$ Department of Applied Mathematics, University of Granada, 18071 Granada, Spain
}

Correspondence should be addressed to D. La Torre; davide.latorre@kustar.ac.ae

Received 3 December 2014; Revised 15 June 2015; Accepted 16 June 2015

Academic Editor: Eric Florentin

Copyright (C) 2015 H. Kunze et al. This is an open access article distributed under the Creative Commons Attribution License, which permits unrestricted use, distribution, and reproduction in any medium, provided the original work is properly cited.

We present an extended version of the Generalized Collage Theorem to deal with inverse problems for vector-valued Lax-Milgram systems. Numerical examples show how the method works in practical cases.

\section{Inverse Problems via the Collage Theorem}

In recent years a great deal of attention has been paid to the inverse problems in distributed systems, that is, the determination of unknown parameters in the functional form of the governing model of the phenomenon under study [14]. The literature is rich in papers studying ad hoc methods to address ill-posed inverse problems by minimizing a suitable approximation error along with utilizing some regularization techniques [5-7].

Many inverse problems may be recast as the approximation of a target element $x$ in a complete metric space $(X, d)$ by the fixed point $\bar{x}$ of a contraction mapping $T$ : $X \rightarrow X$. Thanks to a simple consequence of Banach's fixed point theorem known as the Collage Theorem, most practical methods of solving the inverse problem for fixed point equations seek an operator $T$ for which the collage distance $d(x, T x)$ is as small as possible.

Theorem 1 ("Collage Theorem" [8]). Let $(X, d)$ be a complete metric space and $T: X \rightarrow X$ a contraction mapping with contraction factor $c \in[0,1)$. Then, for any $x \in X$,

$$
d(x, \bar{x}) \leq \frac{1}{1-c} d(x, T x),
$$

where $\bar{x}$ is the fixed point of $T$.
This vastly simplifies this type of inverse problem as it is much easier to estimate $d(x, T x)$ than to find the fixed point $\bar{x}$ and then compute $d(x, \bar{x})$. One now seeks a contraction mapping $T$ that minimizes the so-called collage error $d(x, T x)$, in other words, a mapping that sends the target $x$ as close as possible to itself. This is the essence of the method of collage coding which has been the basis of most, if not all, fractal image coding and compression methods. Barnsley $[8,9]$ was the first to see the potential of using the Collage Theorem above for the purpose of fractal image approximation and fractal image coding. In practical applications, from a family of contraction mappings $T_{\lambda}, \lambda \in \Lambda \subset \mathbb{R}^{n}$, one wishes to find the parameter $\bar{\lambda}$ for which the approximation error $d\left(x, \bar{x}_{\lambda}\right)$ is as small as possible. In practice the feasible set is often taken to be $\Lambda_{c}=\left\{\lambda \in \mathbb{R}^{n}: 0 \leq c_{\lambda} \leq c<1\right\}$ which guarantees the contractivity of $T_{\lambda}$ for any $\lambda \in \Lambda_{c}$. A difference between the "collage" approach and the one based on Tikhonov regularization is the following: in the collage approach, the constraint $\lambda \in \Lambda_{c}$ guarantees that $T_{\lambda}$ is a contraction and, therefore, replaces the effect of the regularization term in the Tikhonov approach (see $[4,7])$. The collage approach works well for low-dimensional parametrization in particular, while Tikhonov regularization is a fundamentally nonparametric methodology. The collage-based inverse 
problem can be formulated as an optimization problem as follows:

$$
\min _{\lambda \in \Lambda_{c}} d\left(x, T_{\lambda} x\right)
$$

This is a nonlinear and nonsmooth optimization model that can often be reduced to a quadratic optimization program. Several algorithms can be used to solve it including penalization methods and particle swarm ant colony techniques.

This method of collage coding may be applied in other situations where contractive mappings are encountered. These ideas have been extended to inverse problems for Initial Value Problems (IVP) in [10]. In this setting, the contractive Picard operator plays the role of $T$ and the space $X$ contains continuous and appropriately bounded functions on a closed interval of observation. Given a target function, perhaps the interpolation of observational data points, the Collage Theorem can be applied to find the Picard operator within a prescribed class that minimizes the collage distance. We have applied this technique to inverse problems involving several families of differential equations and application to different areas (see $[10-15])$.

Example 2. We present the results to an IVP inverse problem solved using collage coding, Tikhonov regularization, and the Landweber-Fridman method. Consider the following steadystate heat equation on $\bar{\Omega}=[0,1]$ :

$$
\begin{aligned}
\frac{d}{d x}\left(\kappa(x) \frac{d u}{d x}(x)\right) & =-f(x), \quad \text { in } \Omega=(0,1), \\
u(0) & =0, \\
u(1) & =0,
\end{aligned}
$$

where $\kappa(x)$ is the variable thermal diffusivity of the medium at $x \in \Omega, f(x)$ represents a heat source or sink at each $x \in \Omega$, and $u(x)$ denotes the temperature at $x \in \Omega$. The inverse problem we look at is to estimate the variable thermal diffusivity $\kappa(x)$ given $r$ uniformly distributed values of $u(x)$, with low-amplitude Gaussian noise added, and the forcing function $f(x)$. For this example, we assume that $u(x)=4 x(1-x)$ and that $f(x)=96 x^{3}+12 x^{2}+48 x-8$, corresponding to $\kappa(x)=\kappa_{\text {True }}(x)=3 x^{3}+2 x^{2}+4 x+1$. Morozov's discrepancy principle was used to find the value of the Tikhonov regularization parameter, with our discrepancy below a tolerance level of $10^{-8}$. Table 1 presents the results; the subscripts indicate the method used to solve the inverse problem.

In a manner analogous to the Collage Theorem we have also formulated a Generalized Collage Theorem for solving Boundary Value Problems (BVP), replacing the minimization of the true error by the minimization of something akin to the collage distance. In place of Banach's fixed point theorem for contraction maps on a complete metric space, we have appealed to the Lax-Milgram representation theorem.
These results have been extended to a wider class of elliptic equations problems in $[16,17]$, by considering not only Hilbert but also reflexive Banach spaces and even replacing the primal variational formulation of such a problem with a more general constrained variational one. Let us mention that this kind of formulation arises, for instance, when the boundary constraints are weakly imposed.

The paper is organized as follows. Section 2 is concerned with an extension of the Collage Theorem stated in [16, Corollary 4.2] to a finite-dimensional vector-valued context, as well as a discretization scheme based on the use of suitable Schauder bases. Section 3 presents three different numerical examples which show how to solve inverse problems for systems of elliptic differential equations.

\section{Vector-Valued Lax-Milgram and the Inverse Problem}

In this section we deal with a Lax-Milgram theorem stated in terms of a system of suitable variational equations and with a collage type result that follows from it. We refer to [18-21] for some recent vectorial versions of the Lax-Milgram theorem and some applications to the study of mixed variational equations.

The first result is the following vector-valued version of the Lax-Milgram theorem, which is a direct consequence of the characterization of the solvability of systems with infinitely many variational equations given in [22, Theorem 3.2] - specifically of its finite-dimensional case [22, Corollary 4.6] - and of the fact that if $N \geq 1, E, F_{1}, \ldots, F_{N}$ are real vector spaces, $a_{1}: E \times F_{1} \rightarrow \mathbb{R}, \ldots, a_{N}: E \times F_{N} \rightarrow \mathbb{R}$ are bilinear forms, and $y_{1}^{*}: F_{1} \rightarrow \mathbb{R}, \ldots, y_{N}^{*}: F_{N} \rightarrow \mathbb{R}$ are linear forms such that the system

$$
x \in E \quad \text { with }\left\{\begin{array}{c}
y_{1}^{*}=a_{1}(x, \cdot) \\
\vdots \\
y_{N}^{*}=a_{N}(x, \cdot)
\end{array}\right.
$$

admits a solution, then such a solution is unique if, and only if, the corresponding homogeneous problem has one and only one solution.

Given a real normed space $G$, we write $G^{*}$ for its topological dual space.

Theorem 3. Suppose that $E$ is a real reflexive Banach space, $N \geq 1, F_{1}, \ldots, F_{N}$ are real Banach spaces, and $a_{1}: E \times F_{1} \rightarrow$ $\mathbb{R}, \ldots, a_{N}: E \times F_{N} \rightarrow \mathbb{R}$ are continuous bilinear forms. Then for all $y_{1}^{*} \in F_{1}^{*}, \ldots, y_{N}^{*} \in F_{N}^{*}$ there exists a unique $x_{0} \in E$ such that

$$
\begin{gathered}
y_{1}^{*}=a_{1}\left(x_{0}, \cdot\right) \\
\vdots \\
y_{N}^{*}=a_{N}\left(x_{0}, \cdot\right)
\end{gathered}
$$


TABLE 1: Recovered diffusivity using collage coding, Tikhonov regularization, and Landweber-Fridman iteration, with $r$ being the number of uniformly distributed sample points in $[0,1], \epsilon$ being the amplitude of the relative noise added, and the true value of $\kappa(x)=1+4 x+2 x^{2}+3 x^{3}$.

\begin{tabular}{|c|c|c|c|}
\hline$r$ & $\epsilon$ & $\kappa_{\text {collage }}(x)$ & $\left\|\kappa_{\text {collage }}-\kappa_{\text {True }}\right\|_{L^{2}(\Omega)}$ \\
\hline \multirow{3}{*}{10} & 0 & $1.0145+3.7757 x+2.7627 x^{2}+2.4460 x^{3}$ & 0.00222 \\
\hline & 0.01 & $1.0129+3.7815 x+2.7685 x^{2}+2.4525 x^{3}$ & 0.00295 \\
\hline & 0.1 & $0.9971+3.8350 x+2.8023 x^{2}+2.5362 x^{3}$ & 0.00175 \\
\hline \multirow{3}{*}{15} & 0 & $1.0145+3.7757 x+2.7627 x^{2}+2.4460 x^{3}$ & 0.00152 \\
\hline & 0.01 & $1.0186+3.7915 x+2.7777 x^{2}+2.4619 x^{3}$ & 0.00276 \\
\hline & 0.1 & $1.0578+3.9525 x+2.8989 x^{2}+2.6151 x^{3}$ & 0.00878 \\
\hline$r$ & $\epsilon$ & $\kappa_{\text {Tikhonov }}(x)$ & $\left\|\kappa_{\text {Tikhonov }}-\kappa_{\text {True }}\right\|_{L^{2}(\Omega)}$ \\
\hline \multirow{3}{*}{10} & 0 & $1.2496+4.3996 x-4.2951 x^{2}+10.924 x^{3}$ & 0.65853 \\
\hline & 0.01 & $1.2489+4.3982 x-4.3003 x^{2}+10.930 x^{3}$ & 0.65772 \\
\hline & 0.1 & $1.2428+4.3863 x-4.3472 x^{2}+10.986 x^{3}$ & 0.65165 \\
\hline \multirow{3}{*}{15} & 0 & $1.0434+6.6156 x-9.5908 x^{2}+14.118 x^{3}$ & 0.59133 \\
\hline & 0.01 & $1.0397+6.6132 x-9.5858 x^{2}+14.054 x^{3}$ & 0.58914 \\
\hline & 0.1 & $1.0351+6.6057 x-9.5614 x^{2}+14.022 x^{3}$ & 0.57156 \\
\hline$r$ & $\epsilon$ & $\kappa_{\text {Landweber }}(x)$ & $\left\|\kappa_{\text {Landweber }}-\kappa_{\text {True }}\right\|_{L^{2}(\Omega)}$ \\
\hline \multirow{3}{*}{10} & 0 & $1.2496+4.3996 x-4.2951 x^{2}+10.924 x^{3}$ & 0.65853 \\
\hline & 0.01 & $1.2490+4.3932 x-4.2856 x^{2}+10.921 x^{3}$ & 0.65812 \\
\hline & 0.1 & $1.2441+4.3362 x-4.2004 x^{2}+10.899 x^{3}$ & 0.65663 \\
\hline \multirow{3}{*}{15} & 0 & $1.0469+6.6156 x-9.5908 x^{2}+14.118 x^{3}$ & 0.59133 \\
\hline & 0.01 & $1.0460+6.6133 x-9.5758 x^{2}+14.105 x^{3}$ & 0.59103 \\
\hline & 0.1 & $1.0377+6.5920 x-9.4409 x^{2}+13.993 x^{3}$ & 0.58968 \\
\hline
\end{tabular}

if, and only if,

$$
\begin{gathered}
x \in E, \\
\left.\begin{array}{c}
y_{1}^{*}=a_{1}\left(x_{0}, \cdot\right) \\
\vdots \\
y_{N}^{*}=a_{N}\left(x_{0}, \cdot\right)
\end{array}\right\} \Longrightarrow x=0
\end{gathered}
$$

and there exists $\rho>0$ satisfying

$$
\begin{aligned}
\left(y_{1}, \ldots, y_{N}\right) & \in F_{1} \times \cdots \times F_{N} \\
& \Longrightarrow \rho \sum_{k=1}^{N}\left\|y_{k}\right\| \leq\left\|\sum_{k=1}^{N} a_{k}\left(\cdot, y_{k}\right)\right\|
\end{aligned}
$$

Moreover, if these equivalent conditions hold and $x_{0} \in E$ is the unique solution, then

$$
\left\|x_{0}\right\| \leq \frac{1}{\rho} \max _{k=1, \ldots, N}\left\|y_{k}^{*}\right\|
$$

The aforementioned generalization of the collage type result [16, Corollary 4.2] for a finite number of variational equations is stated in these terms.

Corollary 4. Let $E$ be a real reflexive Banach space, let $N \geq 1$, let $F_{1}, \ldots, F_{N}$ be real Banach spaces, let $y_{1}^{*} \in F_{1}^{*}, \ldots, y_{N}^{*} \in F_{N}^{*}$, and let $\Lambda$ be a nonempty set such that for all $\lambda \in \Lambda$ there exist
$N$ continuous bilinear forms $a_{1 \lambda}: E \times F_{1} \rightarrow \mathbb{R}, \ldots, a_{N \lambda}:$ $E \times F_{N} \rightarrow \mathbb{R}$ and $\rho_{\lambda}>0$ with

$$
\begin{aligned}
& x \in E, \\
& \left.\begin{array}{c}
y_{1}^{*}=a_{1 \lambda}\left(x_{0}, \cdot\right) \\
\vdots \\
y_{N}^{*}=a_{N \lambda}\left(x_{0}, \cdot\right)
\end{array}\right\} \Longrightarrow x=0 \\
& \left(y_{1}, \ldots, y_{N}\right) \in F_{1} \times \cdots \times F_{N} \\
& \Longrightarrow \rho_{\lambda} \sum_{k=1}^{N}\left\|y_{k}\right\| \leq\left\|\sum_{k=1}^{N} a_{k \lambda}\left(\cdot, y_{k}\right)\right\| .
\end{aligned}
$$

Let us also suppose that, for all $\lambda \in \Lambda, x_{\lambda} \in E$ is the unique solution of the variational system

$$
\begin{gathered}
x \in E, \\
y_{1}^{*}=a_{1 \lambda}(x, \cdot) \\
\vdots \\
y_{N}^{*}=a_{N \lambda}(x, \cdot) .
\end{gathered}
$$

Then for each $x_{0} \in E$ and for all $\lambda \in \Lambda$ the inequality

$$
\left\|x_{\lambda}-x_{0}\right\| \leq \frac{1}{\rho_{\lambda}} \max _{k=1, \ldots, N}\left\|y_{k}^{*}-a_{k \lambda}\left(x_{0}, \cdot\right)\right\|
$$

is valid. 
Proof. The unisolvency and continuous dependence on the initial data of the solution in Theorem 3 imply the announced result. Indeed, let $\lambda \in \Lambda$ and notice that $x_{\lambda}-x_{0}$ is a solution of the system of variational equations

$$
\begin{gathered}
x \in E, \\
y_{1}^{*}-a_{1 \lambda}\left(x_{0}, \cdot\right)=a_{1 \lambda}(x, \cdot) \\
\vdots \\
y_{N}^{*}-a_{N \lambda}\left(x_{0}, \cdot\right)=a_{N \lambda}(x, \cdot) .
\end{gathered}
$$

Then, in view of Theorem 3 , we conclude that

$$
\left\|x_{\lambda}-x_{0}\right\| \leq \frac{1}{\rho_{\lambda}} \max _{k=1, \ldots, N}\left\|y_{k}^{*}-a_{k \lambda}\left(x_{0}, \cdot\right)\right\| .
$$

Let us observe that if one wants to approximate the solution $x_{0}$ in the sense of the collage distance, that is, minimize $\left\{\left\|x_{\lambda}-x_{0}\right\|: \lambda \in \Lambda\right\}$, according to Corollary 4 , it suffices to minimize

$$
\left\{\frac{1}{\rho_{\lambda}} \max _{k=1, \ldots, N}\left\|y_{k}^{*}-a_{k \lambda}\left(x_{0}, \cdot\right)\right\|: \lambda \in \Lambda\right\},
$$

although if

$$
\rho:=\inf _{\lambda \in \Lambda} \rho_{\lambda}>0,
$$

then we only need to minimize

$$
\left\{\max _{k=1, \ldots, N}\left\|y_{k}^{*}-a_{k \lambda}\left(x_{0}, \cdot\right)\right\|: \lambda \in \Lambda\right\} .
$$

Under such an assumption, $\rho>0$, and suppose that each space $F_{k}(k=1, \ldots, N)$ has a Schauder basis $\left\{Y_{k i}\right\}_{i \geq 1}$, in such a way that if $\left\{\Upsilon_{k i}^{*}\right\}_{i \geq 1}$ denotes its sequence of biorthogonal functionals, then the nonrestrictive condition

$$
M:=\max _{k=1, \ldots, N} \sup _{i \geq 1}\left\|\Upsilon_{k i}^{*}\right\|<\infty
$$

holds. In order to discretize our optimization problem, let us also assume that $E$ admits a Schauder basis $\left\{\Theta_{i}\right\}_{i \geq 1}$ and define for each $n \geq 1$ and $k=1, \ldots, N$

$$
\begin{gathered}
E_{n}:=\operatorname{span}\left\{\Theta_{1}, \ldots, \Theta_{n}\right\}, \\
F_{k n}:=\operatorname{span}\left\{\Upsilon_{k 1}, \ldots, \Upsilon_{k n}\right\}
\end{gathered}
$$

and let $P_{n}$ be the $n$ th-projection of $E$ onto $E_{n}$; that is, for all $x \in E$,

$$
P_{n} x:=\sum_{i=1}^{n} \Theta_{i}^{*}(x) \Theta_{i} .
$$

We also suppose that for all $\lambda \in \Lambda, k=1, \ldots, N$, and $n \geq 1$

$$
\begin{gathered}
x \in E_{n}, \\
\left\{\begin{array}{c}
0=a_{1 \lambda}(x, \cdot) \\
\vdots \\
0=a_{N \lambda}(x, \cdot)
\end{array} \Longrightarrow x=0,\right.
\end{gathered}
$$

and there exists $\rho_{\lambda}^{n}>0$ such that

$$
\begin{aligned}
\left(y_{1}, \ldots, y_{N}\right) & \in F_{1 n} \times \cdots \times F_{N n} \\
& \Longrightarrow \rho_{\lambda}^{n} \sum_{k=1}^{N}\left\|y_{k}\right\| \leq\left\|\sum_{k=1}^{N} a_{k \lambda}\left(\cdot, y_{k}\right)\right\| .
\end{aligned}
$$

Then, Theorem 3 guarantees the existence of unique $x_{j}^{n} \in E_{n}$ such that

$$
\begin{aligned}
\left(y_{1}, \ldots y_{N}\right) \in F_{1 n} \times F_{n N} \\
\Longrightarrow\left\{\begin{array}{c}
y_{1}^{*}\left(y_{1}\right)=a_{1}\left(x_{j}^{n}, y_{1}\right) \\
\vdots \\
y_{N}^{*}\left(y_{N}\right)=a_{N}\left(x_{j}^{n}, y_{N}\right) .
\end{array}\right.
\end{aligned}
$$

Corollary 4 , when applied to this vector-valued variational problem, implies

$$
\begin{aligned}
& \left\|x_{\lambda}^{n}-P_{n} x_{0}\right\| \\
& \quad \leq \frac{M}{\rho_{\lambda}^{n}} \max _{k=1, \ldots, N} \sum_{i=1}^{n}\left|y_{0}^{*}\left(\Upsilon_{k i}\right)-a_{k \lambda}\left(P_{n} x_{0}, \Upsilon_{k i}\right)\right|,
\end{aligned}
$$

and if

$$
\gamma:=\sup _{\lambda \in \Lambda, n \geq 1} \rho_{\lambda}^{n}>0
$$

then it suffices to minimize

$$
\varphi_{n}(j):=\max _{k=1, \ldots, N} \sum_{i=1}^{n}\left|y_{0}^{*}\left(\Upsilon_{k i}\right)-a_{k \lambda}\left(P_{n} x_{0}, \Upsilon_{k i}\right)\right|
$$

or equivalently the discrete objective function

$$
\Phi_{n}(j):=\sum_{k=1}^{N} \sum_{i=1}^{n}\left(y_{0}^{*}\left(\Upsilon_{k i}\right)-a_{k \lambda}\left(P_{n} x_{0}, \Upsilon_{k i}\right)\right)^{2},
$$

which is easier to minimize.

\section{Numerical Examples}

In this section we present three different numerical examples.

Example 1. We consider the linear system

$$
\begin{aligned}
-\frac{d}{d x}\left(\kappa(x) \frac{d u}{d x}\right)+A u & =f(x), \quad 0<x<1, \\
u(0) & =0, \\
u(1) & =0
\end{aligned}
$$

with

$$
\begin{gathered}
\kappa(x)=5+2 x, \\
u=\left[\begin{array}{l}
u_{1} \\
u_{2}
\end{array}\right], \\
A=3, \\
f(x)=\left[\begin{array}{l}
f_{1}(x) \\
f_{2}(x)
\end{array}\right]=\left[\begin{array}{c}
8 x \\
1
\end{array}\right] .
\end{gathered}
$$




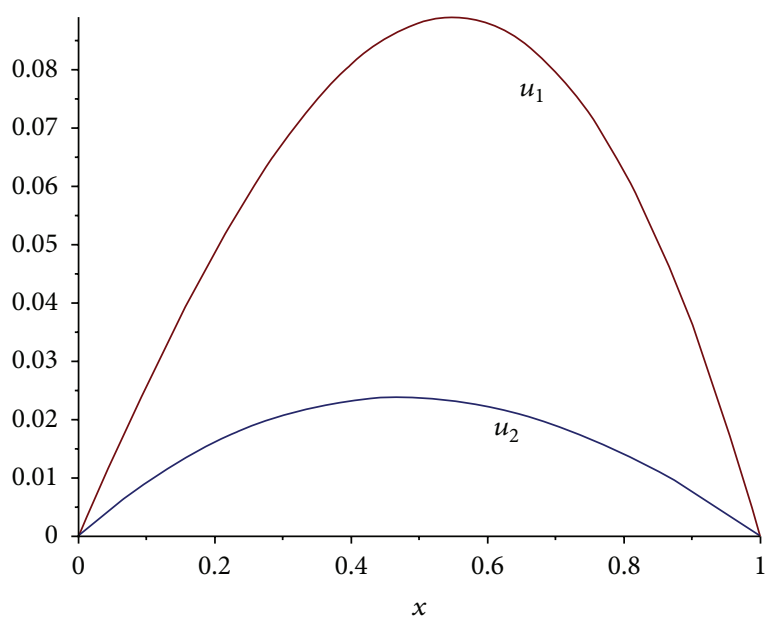

(a)

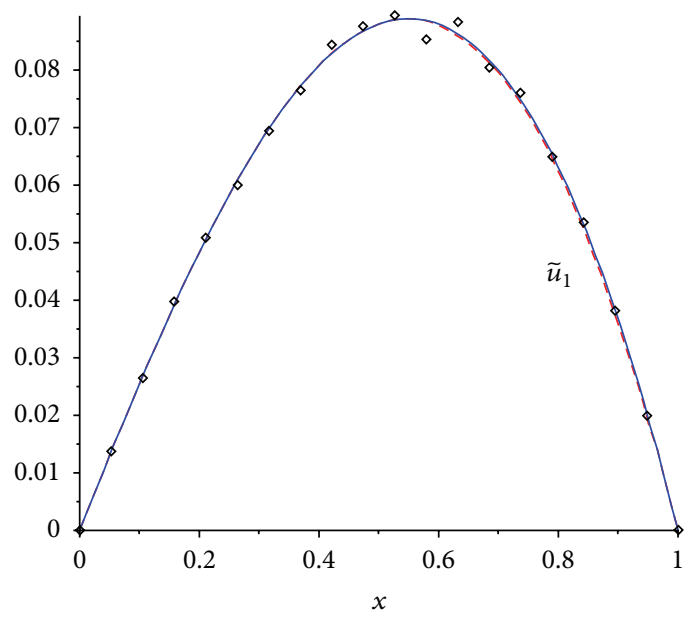

(b)

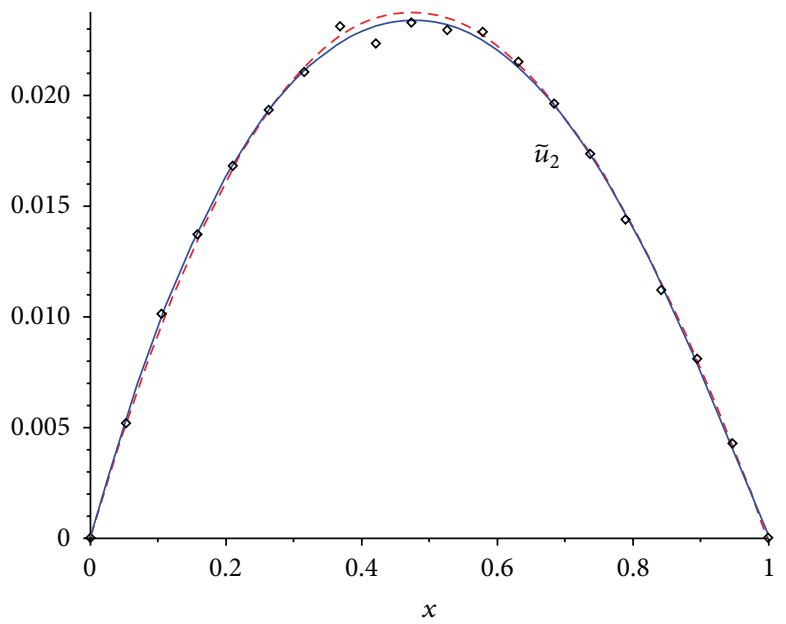

(c)

FIgURe 1: For Example 1: (a) the numerical solution $u$, (b) the noised target $\widetilde{u}_{1}$, and (c) the noised target $\widetilde{u}_{2}$, with $5 \%$ relative noise added.

We solve the linear system, sample each solution component at $M$ uniformly distributed data points in $[0,1]$, add relative noise of $\varepsilon$, and fit 6th-degree polynomials to the resulting data to produce a target solution $\widetilde{u}$. Figure 1 shows the numerical solution $u$ and the target functions $\widetilde{u}_{i}$ with $M=20$ and $\epsilon=0.05$. We consider the inverse problem: Given $\tilde{u}$ and $f(x)$, approximate $\kappa(x)$ and the coefficient $A$ such that the resulting system admits $\tilde{u}$ as an approximate solution. We set

$$
\kappa(x)=\sum_{i=1}^{N} \lambda_{i} x^{i} .
$$

With $\left\{\xi_{i}\right\}_{i=1}^{n}$ equal to the $n$-dimensional "hat basis" of $[0,1]$, for various values of $M$, noise $\varepsilon, \kappa$ degree $N$, and basis dimension $n$, we construct the objective function in (26) and minimize it to find $\lambda=\left(A, \lambda_{0}, \ldots, \lambda_{N}\right)$. The results are presented in Table 2.
Example 2. We consider the coupled system

$$
\begin{aligned}
-\frac{d}{d x}\left(\kappa(x) \frac{d u}{d x}\right)+L u & =f(x) \quad 0<x<1, \\
u(0) & =0, \\
u(1) & =0,
\end{aligned}
$$

with

$$
\begin{gathered}
\kappa(x)=5+2 x, \\
L=\left[\begin{array}{cc}
0 & 2 \\
-3 & 0
\end{array}\right],
\end{gathered}
$$

$$
f(x)=\left[\begin{array}{l}
f_{1}(x) \\
f_{2}(x)
\end{array}\right]=\left[\begin{array}{c}
x^{-3 / 5} \\
(1-x)^{-3 / 5}
\end{array}\right] .
$$

Note that the forcing functions $f_{1}(x)$ and $f_{2}(x)$ are not Hilbertian, living instead in $W_{0}^{1,3 / 2}(0,1)$, for example. We 
TABLE 2: Recovered parameter values for Example 1. True values are $\lambda=(3,5,2,0)$.

\begin{tabular}{lcccc}
\hline$M$ & $n$ & $\varepsilon$ & $N=1$ & \multicolumn{2}{c}{ Recovered $\lambda$} \\
& & & $(3.0000,4.9999,2.0001,0.0001)$ \\
10 & 20 & 0 & $(3.0000,4.9999,2.0002)$ & $(3.0259,4.9717,1.7660,0.3278)$ \\
10 & 20 & 0.01 & $(2.9628,4.9411,2.1213)$ & $(3.0879,4.9132,1.2916,0.9902)$ \\
10 & 20 & 0.03 & $(2.9108,4.8174,2.3647)$ & $(3.1637,4.8520,0.8091,1.6614)$ \\
10 & 20 & 0.05 & $(3.0001,4.9998,2.0003)$ & $(3.0001,4.9998,2.0002,0.0001)$ \\
30 & 40 & 0 & $(2.9250,4.9758,2.0816)$ & $(3.0265,5.0203,1.5585,0.4778)$ \\
30 & 40 & 0.01 & $(2.8888,4.8936,2.2519)$ & $(3.1537,5.0431,0.6498,1.4600)$ \\
30 & 40 & 0.05 & $(3.0083,4.7664,2.4290)$ & $(3.3781,5.0415,-0.2832,2.4671)$ \\
30 & 40 & & &
\end{tabular}

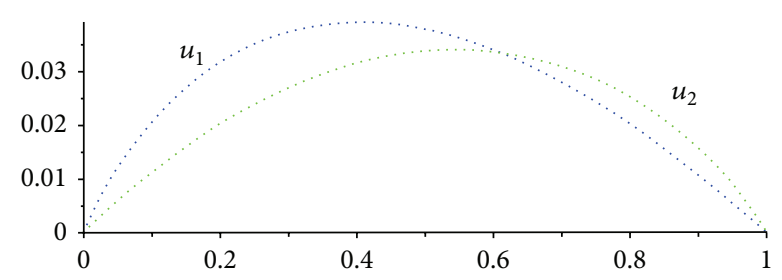

FIgURE 2: The numerical solution at 511 points for Example 2.

work in the reflexive framework of Section 2. (In [16], we observed that the Hilbert space solution framework failed to work for a single equation with forcing function $f_{1}(x)$.) Following [23, Proposition 4.8], we can construct a Schauder basis $\left\{e_{n}\right\}_{n \in \mathbb{N}}$ in the Sobolev space $W_{0}^{1, p}(0,1)$ by integrating the Haar system of $L_{p}(0,1)$.

The BVP has singularities at $x=0$ and $x=1$, since at each endpoint one of $f_{1}(x)$ or $f_{2}(x)$ is undefined. The forward problem can be solved numerically by using collocation techniques. We use COMSOL to solve it. Figure 2 presents the numerical solution. Next, we represent the solution in the subspace $E_{511}$ generated by the first 511 terms of the Schauder basis; call these representations $\widetilde{u}_{1}(x)$ and $\widetilde{u}_{2}(x)$. We consider the inverse problem: find $K(x)$ such that $\tilde{u}$ is admitted as an approximate solution to (30) with $\kappa(x)$ replaced by $K(x)$.

We solve the inverse problem by constructing the objective function in (26) with $n=511, \xi_{i}$ equal to the $i$ th element of the Schauder basis, and $K(x)=\sum_{i=0}^{N} \lambda_{i} x^{i}$. With these choices, we have that

$$
\begin{aligned}
\left(a_{1}\right)_{\lambda}\left(\tilde{u}, \xi_{i}\right)= & \int_{0}^{1}\left(K(x) \xi_{i}^{\prime}(x) \tilde{u}_{1}^{\prime}(x)\right) d x \\
& +2 \int_{0}^{1} \widetilde{u}_{2}(x) \xi_{i}(x) d x \\
\left(a_{2}\right)_{\lambda}\left(\widetilde{u}, \xi_{i}\right)= & \int_{0}^{1}\left(K(x) \xi_{i}^{\prime}(x) \tilde{u}_{2}^{\prime}(x)\right) d x \\
& -3 \int_{0}^{1} \widetilde{u}_{1}(x) \xi_{i}(x) d x \\
\left(\left(T_{0}\right)_{1}\right)_{\lambda}\left(\xi_{i}\right)= & \int_{0}^{1} f_{i}(x) \xi_{i}(x) d x .
\end{aligned}
$$

When $N=1$ upon minimizing the objective function we obtain, to four decimal places, $\lambda_{0}=5.0893$ and $\lambda_{1}=1.7920$. When $N=2$, we find $\lambda_{0}=5.0698, \lambda_{1}=1.9840$, and $\lambda_{2}=-0.1929$.

\section{Example 3. We consider the 2D linear system}

$$
\begin{aligned}
-\nabla \cdot(\kappa(x, y) \nabla u)+A u & =f(x, y), \\
\quad 0<x<1,0<y<1, & \\
u(0, y) & =0, \\
u(1, y) & =0, \\
u(x, 0) & =0, \\
u(x, 1) & =0,
\end{aligned}
$$

with

$$
\begin{gathered}
\kappa(x)=\left[\begin{array}{cc}
10+2 x+3 y & 0 \\
0 & 10+2 x+3 y
\end{array}\right], \\
u=\left[\begin{array}{l}
u_{1} \\
u_{2}
\end{array}\right], \\
A=\left[\begin{array}{ll}
-1 & 3 \\
-2 & 4
\end{array}\right], \\
f(x)=\left[\begin{array}{l}
f_{1}(x) \\
f_{2}(x)
\end{array}\right],
\end{gathered}
$$

where $f_{1}(x, y)$ and $f_{2}(x, y)$ have been chosen so that the actual solution to the system is

$$
u=\left[\begin{array}{l}
\frac{1}{4} x y(1-x)(1-y) \\
\frac{1}{8} x y(1-x)(1-y)
\end{array}\right] .
$$

Analogous to the process followed in Example 1, we sample each solution component at $M \times M$ uniformly distributed data points in $[0,1] \times[0,1]$ and add relative noise of $\varepsilon$ to each of these data points. A target solution $\widetilde{u}$ is constructed 
TABLE 3: Recovered parameter values for Example 3. True values are $\lambda=\gamma=(10,2,3,0)$.

\begin{tabular}{lccc}
\hline$M$ & $\varepsilon$ & Recovered $\lambda$ & Recovered $\gamma$ \\
\hline 10 & 0 & $(9.9282,1.9728,2.9134,0.0068)$ & $(9.9242,1.9264,2.9625,-0.0068)$ \\
10 & 0.01 & $(9.9268,1.9641,2.9096,-0.0097)$ & $(9.9233,1.9257,2.9594,-0.0087)$ \\
10 & 0.03 & $(9.9246,1.9653,2.9065,-0.0157)$ & $(9.9219,1.9218,2.9573,-0.0127)$ \\
10 & 0.05 & $(9.9232,1.9685,2.7664,-0.0221)$ & $(9.9574,1.9919,2.9878,0.0001)$ \\
20 & 0 & $(9.9581,1.9899,2.9921,-0.0018)$ & $(9.9565,1.9827,2.9867,-0.0018)$ \\
20 & 0.01 & $(9.9575,1.9886,2.9780,0.0002)$ & $(9.9456,1.9241,2.9801,0.0063)$ \\
20 & 0.03 & $(9.9481,1.9814,2.9786,0.0065)$ & $(9.9228,1.9152,2.9680,0.0153)$ \\
20 & 0.05 & $(9.9378,1.9682,2.9141,0.0156)$ & $(9.9954,1.9984,2.9957,0.0000)$ \\
30 & 0 & $(10.004,1.9954,2.9904,0.0000)$ & $(9.9761,1.9830,3.0148,-0.0008)$ \\
30 & 0.01 & $(9.9796,1.9965,2.9809,-0.0008)$ & $(9.9656,1.9816,2.9944,-0.0059)$ \\
30 & 0.03 & $(9.9663,2.0146,3.0216,-0.0059)$ & $(9.9640,2.0321,3.0554,-0.0219)$ \\
\hline
\end{tabular}

using these data points together with our basis functions, $\xi_{i j}$ (hexagonal-based pyramids in this $2 \mathrm{D}$ case):

$$
\begin{aligned}
& u_{1}(x, y)=\sum_{i=1}^{M} \sum_{j=1}^{M}\left(u_{1}\right)_{i j} \xi_{i j}, \\
& u_{2}(x, y)=\sum_{i=1}^{M} \sum_{j=1}^{M}\left(u_{2}\right)_{i j} \xi_{i j},
\end{aligned}
$$

$$
\text { for } i, j=1, \ldots, M \text {. }
$$

We consider the inverse problem: Given $\tilde{u}, A$, and $f(x, y)$, approximate $\kappa(x, y)$ such that the resulting system admits $\tilde{u}$ as an approximate solution. We set

$$
\kappa(x, y)=\left[\begin{array}{c}
\sum_{i=0}^{p} \sum_{j=0}^{q} \lambda_{i j} x^{i} y^{j} \\
\sum_{i=0}^{p} \sum_{j=0}^{q} \gamma_{i j} x^{i} y^{j}
\end{array}\right] .
$$

For various values of $M$, noise $\varepsilon, \kappa$ degree 1 , and basis dimension $n=M$, we construct the objective function in (26) and minimize it to find $\lambda_{i j}, \gamma_{i j}$, for $i=1, \ldots, p$ and $j=1, \ldots, q$. The results are presented in Table 3 .

\section{Conflict of Interests}

The authors declare that there is no conflict of interests regarding the publication of this paper.

\section{References}

[1] A. Kirsch, An Introduction to the Mathematical Theory of Inverse Problems, Springer, New York, NY, USA, 2011.

[2] F. D. M. Neto and A. J. da Silva Neto, An Introduction to Inverse Problems with Applications, Springer, New York, NY, USA, 2013.

[3] A. Tarantola, Inverse Problem Theory and Methods for Model Parameter Estimation, SIAM, Philadelphia, Pa, USA, 2005.

[4] C. R. Vogel, Computational Methods for Inverse Problems, vol. 23 of Frontiers in Applied Mathematics, SIAM, New York, NY, USA, 2002.
[5] J. Milstein, "The inverse problem: estimation of kinetic parameters," in Modeling of Chemical Reaction Systems, K. Ebert, P. Deuflhard, and W. Jäger, Eds., Springer Series in Chemical Physics, pp. 92-101, Springer, Berlin, Germany, 1981.

[6] A. N. Tychonoff and V. Y. Arsenin, Solution of Ill-Posed Problems, Winston \& Sons, Washington, DC, USA, 1977.

[7] A. N. Tychonoff, "Solution of incorrectly formulated problems and the regularization method," Doklady Akademii Nauk SSSR, vol. 15, pp. 501-504, 1963.

[8] M. F. Barnsley, Fractals Everywhere, Academic Press, New York, NY, USA, 1989.

[9] M. F. Barnsley, V. Ervin, D. Hardin, and J. Lancaster, "Solution of an inverse problem for fractals and other sets," Proceedings of the National Academy of Sciences of the United States of America, vol. 83, no. 7, pp. 1975-1977, 1986.

[10] H. E. Kunze and E. R. Vrscay, "Solving inverse problems for ordinary differential equations using the Picard contraction mapping," Inverse Problems, vol. 15, no. 3, pp. 745-770, 1999.

[11] H. Kunze, D. la Torre, F. Mendivil, and E. R. Vrscay, FractalBased Methods in Analysis, Springer, 2012.

[12] H. Kunze, D. La Torre, and E. R. Vrscay, "Solving inverse problems for DEs using the collage theorem and entropy maximization," Applied Mathematics Letters, vol. 25, no. 12, pp. 2306-2311, 2012.

[13] H. E. Kunze, D. La Torre, and E. R. Vrscay, "Random fixed point equations and inverse problems using 'collage method' for contraction mappings," Journal of Mathematical Analysis and Applications, vol. 334, no. 2, pp. 1116-1129, 2007.

[14] H. Kunze, D. La Torre, and E. R. Vrscay, "A generalized collage method based upon the Lax-Milgram functional for solving boundary value inverse problems," Nonlinear Analysis. Theory, Methods \& Applications, vol. 71, no. 12, pp. el337-e1343, 2009.

[15] V. Capasso, H. E. Kunze, D. La Torre, and E. R. Vrscay, "Solving inverse problems for differential equations by a 'generalized collage' method and application to a mean field stochastic model," Nonlinear Analysis: Real World Applications, vol. 15, pp. 276-289, 2014

[16] M. I. Berenguer, H. Kunze, D. La Torre, and M. Ruiz Galán, "Galerkin schemes and inverse boundary value problems in reflexive Banach spaces," Journal of Computational and Applied Mathematics, vol. 275, pp. 100-112, 2015.

[17] M. I. Berenguer, H. E. Kunze, D. La Torre, and M. Ruiz Galán, "A collage-based approach to inverse problems for constrained 
variational equations," Journal of Computational and Applied Mathematics. Accepted.

[18] V. V. Semenov, "Projection theorem for Banach and locally convex spaces," Cybernetics and Systems Analysis, vol. 44, no. 5, pp. 722-728, 2008.

[19] D. Boffi, F. Brezzi, and M. Fortin, Mixed Finite Element Methods and Applications, vol. 44 of Springer Series in Computational Mathematics, Springer, Heidelberg, Germany, 2013.

[20] G. N. Gatica, A Simple Introduction to the Mixed Finite Element Method. Theory and Applications, Springer Briefs in Mathematics, Springer, 2014.

[21] A. I. Garralda-Guillem and M. Ruiz Galán, "Mixed variational formulations in locally convex spaces," Journal of Mathematical Analysis and Applications, vol. 414, no. 2, pp. 825-849, 2014.

[22] M. R. Galán, "An intrinsic notion of convexity for minimax," Journal of Convex Analysis, vol. 21, no. 4, pp. 1105-1139, 2014.

[23] S. Fučik, "Fredholm alternative for nonlinear operators in Banach spaces and its applications to differential and integral equations," Časopis pro Pěstování Matematiky, vol. 96, pp. 371390, 1971. 


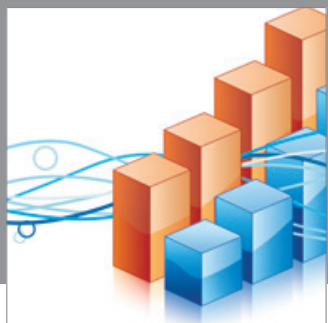

Advances in

Operations Research

mansans

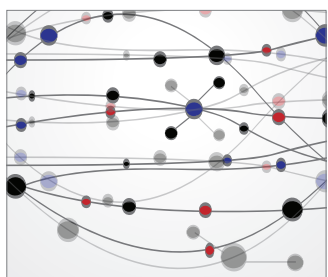

The Scientific World Journal
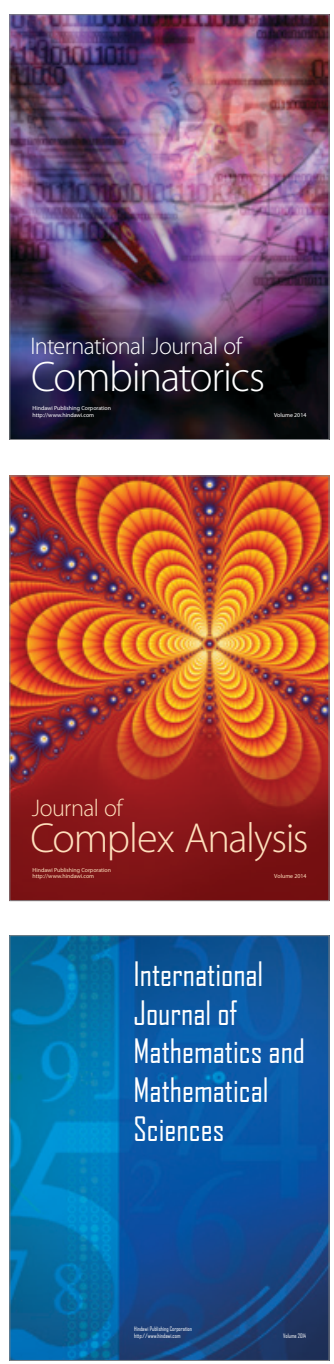
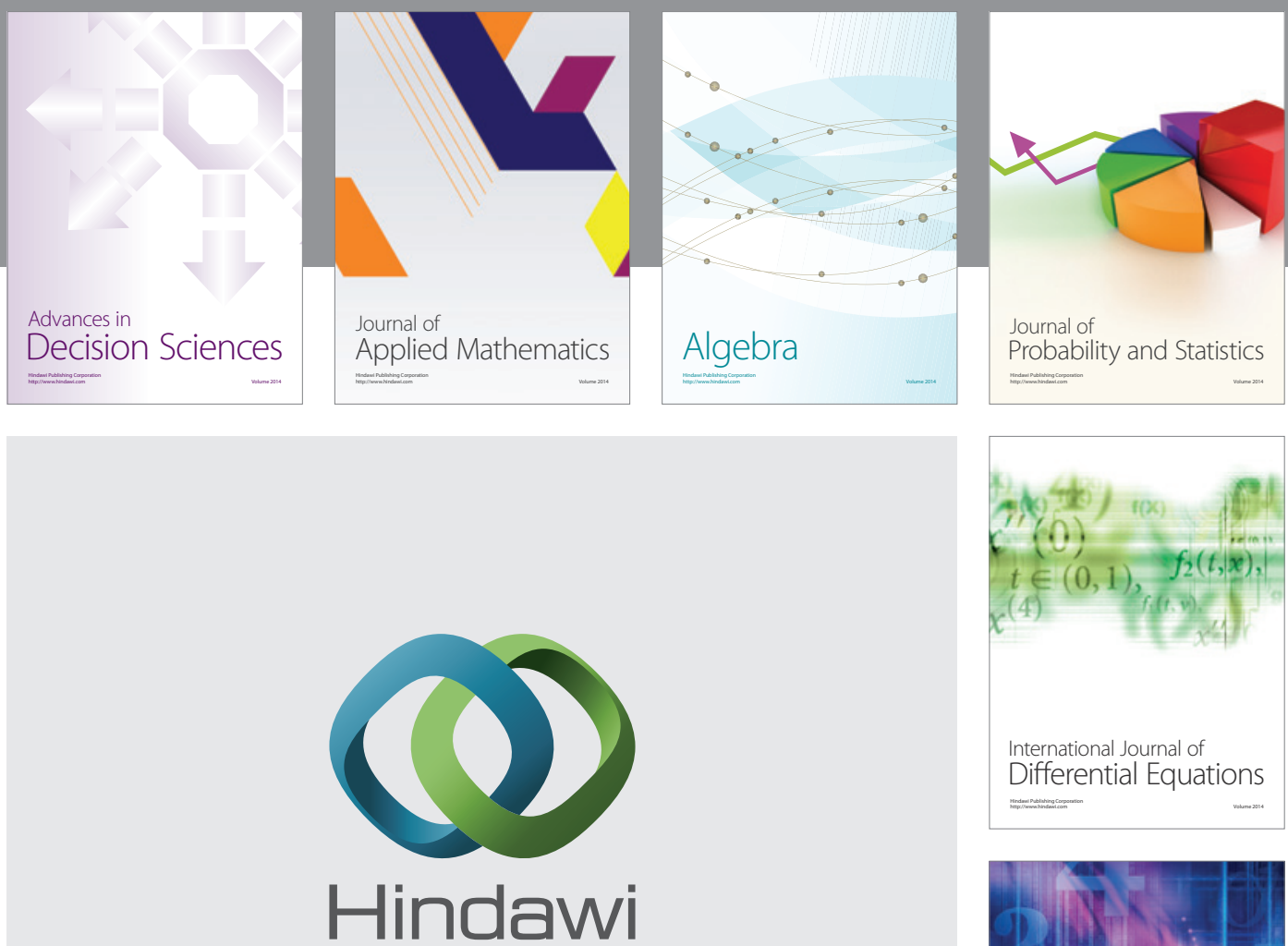

Submit your manuscripts at http://www.hindawi.com
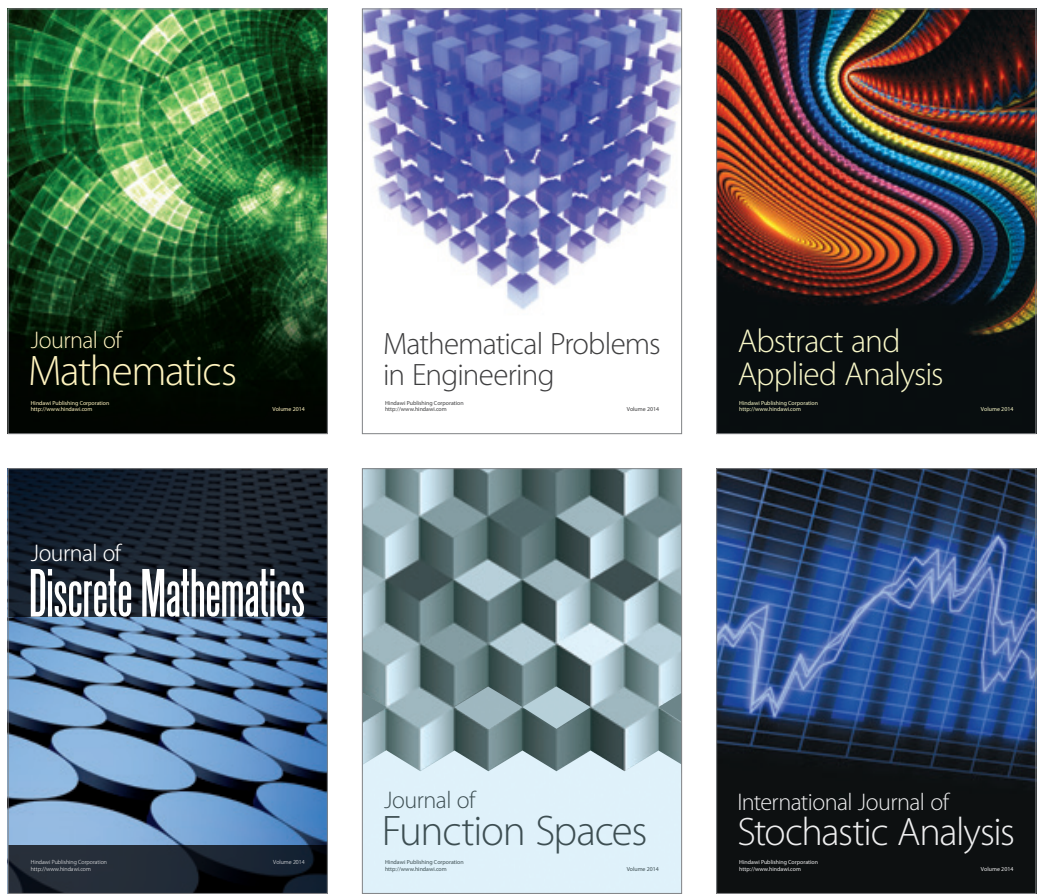

Journal of

Function Spaces

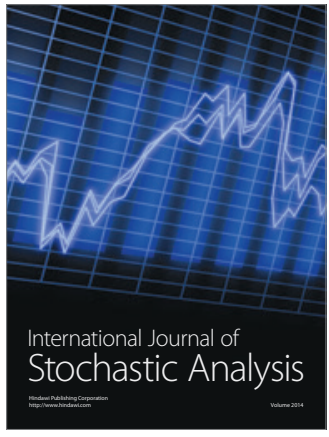

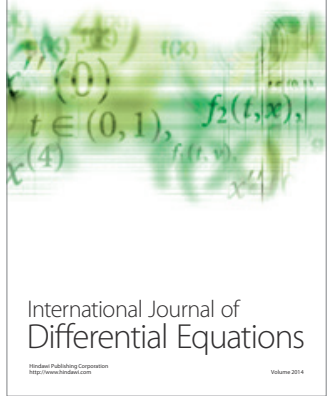
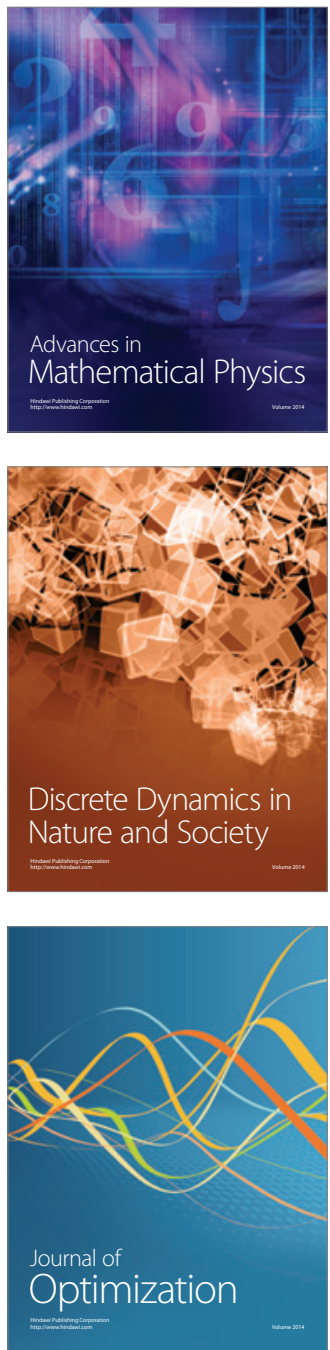